\title{
Two recessive mutations in FGF5 are associated with the long-hair phenotype in donkeys
}

\author{
Romain Legrand, Laurent Tiret and Marie Abitbol ${ }^{*}$
}

\begin{abstract}
Background: Seven donkey breeds are recognized by the French studbook. Individuals from the Pyrenean, Provence, Berry Black, Normand, Cotentin and Bourbonnais breeds are characterized by a short coat, while those from the Poitou breed (Baudet du Poitou) are characterized by a long-hair phenotype. We hypothesized that loss-of-function mutations in the FGF5 (fibroblast growth factor 5) gene, which are associated with a long-hair phenotype in several mammalian species, may account for the special coat feature of Poitou donkeys. To the best of our knowledge, mutations in FGF5 have never been described in Equidae.

Methods: We sequenced the FGF5 gene from 35 long-haired Poitou donkeys, as well as from a panel of 67 short-haired donkeys from the six other French breeds and 131 short-haired ponies and horses.

Results: We identified a recessive c.433_434delAT frameshift deletion in FGF5, present in Poitou and three other donkey breeds and a recessive nonsense c.245G > A substitution, present in Poitou and four other donkey breeds. The frameshift deletion was associated with the long-hair phenotype in Poitou donkeys when present in two copies $(n=31)$ or combined with the nonsense mutation $(n=4)$. The frameshift deletion led to a stop codon at position 159 whereas the nonsense mutation led to a stop codon at position 82 in the FGF5 protein. In silico, the two truncated FGF5 proteins were predicted to lack the critical $\beta$ strands involved in the interaction between FGF5 and its receptor, a mandatory step to inhibit hair growth.

Conclusions: Our results highlight the allelic heterogeneity of the long-hair phenotype in donkeys and enlarge the panel of recessive FGF5 loss-of-function alleles described in mammals. Thanks to the DNA test developed in this study, breeders of non-Poitou breeds will have the opportunity to identify long-hair carriers in their breeding stocks.
\end{abstract}

\section{Background}

The hair growth cycle in mammals is composed of three phases: anagen, when follicles grow and hair is synthetized; catagen, when follicles regress; and telogen, when follicles are inactive [1]. Hair growth is regulated by several cytokines and growth factors, including members from the fibroblast growth factors family (FGF) and their receptors. Eighteen mammalian FGF have been described that exhibit a variety of activities, among which paracrine factors that are involved in tissue patterning and organogenesis during embryogenesis and endocrine factors that are involved in metabolism homeostasis during adulthood [2]. Among the members of the FGF family, FGF5 was first described as a human oncogene [3] and was

\footnotetext{
* Correspondence: m.abitbol@vet-alfort.fr UMR955 INRA-ENVA de Génétique Fonctionnelle et Médicale, Université Paris-Est, Institut National de la Recherche Agronomique, Ecole nationale vétérinaire d'Alfort, F-94700 Maisons-Alfort, France
}

later shown to be involved in the dual-mode regulation of the hair growth cycle $[4,5]$. In rats and mice, alternative splicing of Fgf5 transcripts results in a full-length form of FGF5 and a short form that lacks the sequences encoded by exon 2 and most of exon 3 [1,6]. Functionally, it was demonstrated that the full-length FGF5 induces catagen, a function that is antagonized by the short form during anagen [5]. Indeed, the angora mouse mutant carries a 2-kb loss-of-function deletion in Fgf5 and displays a delayed catagen onset and excessively long truncal hair [7]. Loss-of-function mutations associated with long-hair phenotypes have been described in several mammalian species including mice $[4,8]$, dogs $[9,10]$ and cats $[11,12]$, which support a conserved role of FGF5 in the regulation of hair growth.

Among Equidae, the Poitou donkey breed (Baudet du Poitou) displays a long-hair coat. This breed originated from the Poitou region of France and was originally 
developed to produce a large type of working mules with the Poitevin Mulassier draft horse. The exact origin of the Poitou breed is unknown, probably dating back to before 1884 when the studbook was created in France. During the eighteenth and nineteenth centuries, thousands of Poitou mules were produced and exported to Europe. After World War II, draft animals were replaced by engines and mule production began to drop off. In 1977, the breed was endangered with only 44 Poitou donkeys (20 males and 24 females) registered in France. Thus, an efficient breeding program was set up to save the breed, which led to the reconstitution of a larger breeding stock. In 2005, 82 jacks and 355 jennets were used in breeding schemes (http://www.france-trait.fr).

Poitou is recognized as a large-sized donkey breed with a long-hair coat. When Poitou donkeys are left ungroomed, their coat retains very long hair that becomes matted and tangled and then grows down into a great coat. We hypothesized that the long-hair phenotype in the Poitou breed could result from loss-of-function mutations in the FGF5 gene. Here, we report the precise identification of two loss-of-function alleles in FGF5 that are fully associated with the long-hair phenotype in the panel of animals analysed when they are present as composite or homozygous recessive mutations.

\section{Methods}

\section{Animals and ethics statement}

One hundred and two donkeys from seven breeds were included in the study. They were all sampled in France from September 2012 to October 2013 and originated from Poitou $(n=35)$, Bourbonnais $(n=2)$, Cotentin $(n=10)$, Berry Black $(n=8)$, Normand $(n=18)$, Provence $(n=16)$ and Pyrenean $(\mathrm{n}=13)$ breeds.

All the animals anlysed were included at the owner's request. Pictures, buccal swabs and hair samples were sent directly by the owners or collected by a certified veterinarian (MA).

DNA samples from 131 horses and ponies previously collected for DNA identification purposes were used as controls. The panel included 40 thoroughbred, 33 French saddlebred, 26 Arab horses and eight Connemara, one Icelandic, seven Haflinger, seven French, three Pottok and six Shetland ponies. All animals were client-owned animals, which underwent no invasive sampling procedure, thus, no authorization for animal experiment was required according to the legal definition in Europe (Subject $5 \mathrm{f}$ of Article1, Chapter I of the Directive 2010/63/UE of the European Parliament and of the Council).

\section{DNA extraction}

DNA was extracted from buccal swabs or hair roots using a Maxwell ${ }^{\circledR} 16$ Instrument (Promega Corporation, Madison, USA), according to the manufacturer's protocols.

\section{Sequencing and genotyping of FGF5}

When this project was initiated, the donkey genome sequence was not yet released [13]. Thus, reference genome sequences were based on the equine genome sequence (Equine FGF5 gene, [Ensembl:ENSECAG00000015710]). PCR and sequencing primers were designed using Primer3 [14]. Exon 1 was amplified using FGF5 exon1 $\mathrm{F}$ 5'-AGCGCCGAGATCCGTTC-3' and FGF5 exon1 R 5'-GGACGGGTTTTGGAGGAG-3' primers. Exon 2 was amplified using FGF5 exon2F 5'-TGCAGTAATAAAGA ATGGGAAG-3' and FGF5 exon2R 5' -TGCATTCCATT CTACAAACG-3' primers. Exon 3 was amplified using FGF5 exon3F 5' -GCCCATGGAATTCTTGGTTC-3' and FGF5 exon3R 5'-GCTGAAGCTGTGTCCAAAAGTG-3' primers. A more detailed description of PCR and sequencing primers is provided in Additional file 1: Table S1. PCR amplicons were sequenced in both forward and reverse directions using the Sanger method (GATC Biotech AG, Konstanz, Germany), and electropherograms were manually inspected with Chromas Lite (Technelysium Pty Ltd, South Brisbane, Australia). Multiple alignments were performed using Multalin [15].

The genotype of the FGF5 c.433_434delAT deletion was determined using a pyrosequencing method adapted from Ahmadian et al. [16] on a PyroMark Q96 ID pyrosequencer (Qiagen, Hilden, Germany) and the following forward and reverse PCR primers for FGF5 c.433-434delAT: 5' -AATACGAGGAGTTTTCAGCAA CAA-3' and 5'-BIOTIN-CTTGCATGGAGTTTTCC TTTTT-3', and the sequencing primer $5^{\prime}$-AGCAAC AAATTTTTAGCG-3'.

The genotype of the FGF5 c.245G > A SNP was determined using the same method and the following forward and reverse PCR primers :5' -TTGGAGCAGGGCAGTT TC-3' and 5' -BIOTIN-TTGCCATCCGGGTAGATC -3' and the sequencing primer 5'-CAGGGCAGTTTCCAG-3'. A more detailed description of genotyping primers is provided in Additional file 1: Table S2.

\section{Comparison of protein sequences and structure prediction}

The Ensembl sequence of equine FGF5 was used [Ensembl:ENSECAP00000013338]. Multiple alignments were performed using Multalin [15]. The potential negative impact of missense mutations was assessed using the HumVar-trained version of PolyPhen-2. This version is designed to distinguish between mutations with drastic effects and other variations including abundant mildly deleterious alleles [17]. Because the 3D-structure of FGF5 has not been experimentally resolved, homology modeling and fold recognition were performed using the Phyre server [18] and human FGF1, FGF2, FGF9, FGF10 and FGF20 as templates. 


\section{Accession numbers}

Genomic coding sequences of FGF5 from Berry Black (short-hair) and Poitou (long-hair) donkeys (Equus asinus) were submitted to GenBank. Accession numbers are [GenBank: KJ725176] for the wild type short-hair allele, [GenBank: KJ725177] for the c.433_434delAT allele and [GenBank: KJ725178] for the c.245G > A allele.

\section{Results}

The long-hair phenotype, which is very common in some domestic animals such as dogs and cats, is seldom observed in Equidae with the exception of the French Poitou donkey breed. Poitou donkeys are characterized by their large size and shaggy coat that is caused by long, soft hair arranged in long cords when ungroomed (Figure 1). Segregation analysis showed that long-hair is a recessive trait in donkeys. Indeed, F1 Poitou crossbred donkeys and mules are always short-haired. With the aim of providing breeders with an accurate DNA-based test to detect long-hair carriers in Poitou crossbred descendants, we performed a mutation screening of FGF5 in various donkey breeds. Apart from comparative cytogenetic maps, whole-genome mapping tools are still lacking for this species $[19,20]$. Indeed, the SNP (single nucleotide polymorphism) array that was developed for the horse genome [21,22] is of little use for domestic donkeys, with a cross-species validation rate of $5.6 \times 10^{-3}$ for the 53k SNP array [22]. Thus, to circumvent these limitations, we screened long-haired donkeys for mutations in the FGF5 gene, which is the most relevant candidate gene to explain this variation in coat length. To cover the three exons and their respective intron-exon flanking sequences, we designed three sets of intron primers using the equine FGF5 Ensembl sequence.

First, a panel composed of three long-haired Poitou donkeys and two short-haired Berry Black control donkeys was used. The three sets of primers yielded amplicons at the right size, and sequencing confirmed that FGF5 sequences were successfully amplified. Global alignement revealed $99.7 \%$ similarity between the equine and donkey FGF5 coding sequences, which confirmed that the two genes are orthologous. Furthermore, pairwise base-to-base comparisons of sequences between donkeys and the horse reference sequence revealed two interspecific variants, one SNP and a two-base pair deletion from the individuals of this first panel (Table 1). Careful analysis of the two interspecific variants revealed that they consisted of a synonymous mutation and a non-synonymous mutation, which based on PolyPhen-2 prediction has no impact on FGF5 function (L223S; score 0.00$)$. On the contrary, the donkey SNP is a nonsense mutation (c.245G > A) that leads to a stop codon at position 82 in FGF5 and the two-base pair deletion (c.433_434delAT) produces a frameshift that results in a stop codon at position 159 in FGF5 (Table 1).

In a second series of experiments, the complete cohort composed of 102 donkeys representing seven breeds was genotyped for the c.245G > A SNP and the

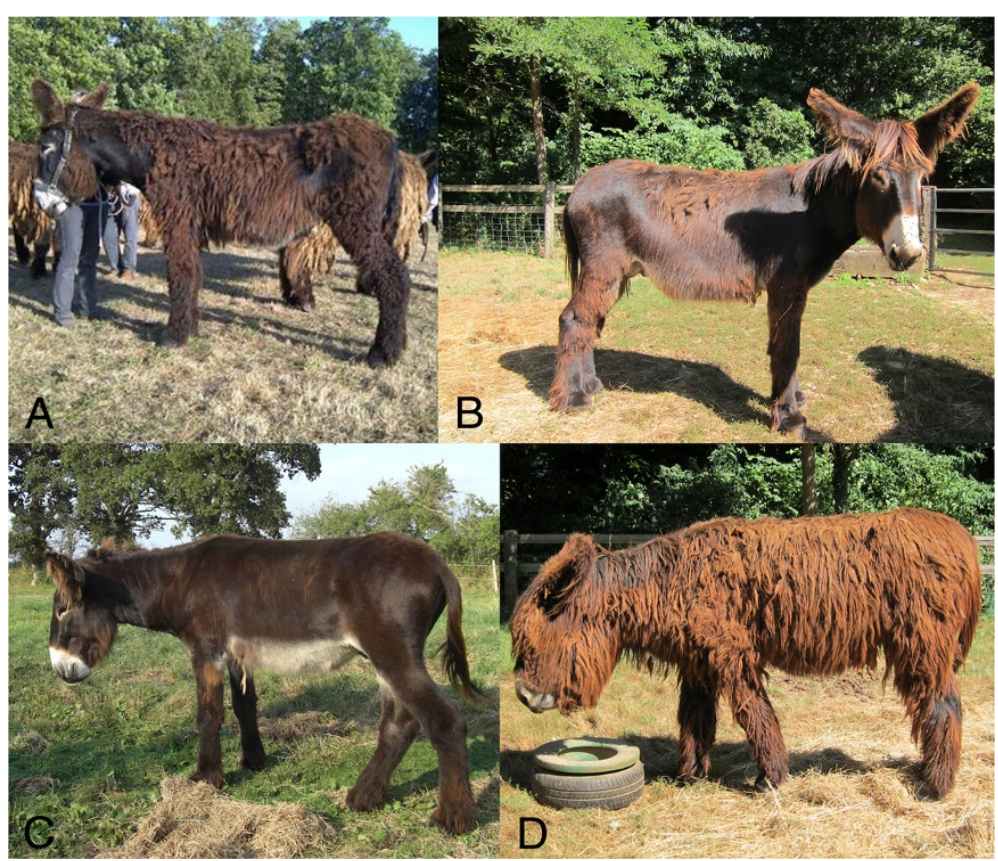

Figure 1 Long-hair phenotype in Poitou donkeys. Poitou donkeys exhibit lifelong hair growth with young animals usually showing a medium-sized coat with short-hair areas (A, B, C) and adult donkeys showing a long matted and tangled coat (D). 
Table 1 FGF5 polymorphisms in donkey exon sequences compared to the horse reference coding sequence

\begin{tabular}{|c|c|c|c|c|}
\hline & $\begin{array}{l}\text { c. } 245 G>A \\
\text { exon } 1\end{array}$ & $\begin{array}{l}\text { c.433_434delAT } \\
\text { exon } 2\end{array}$ & $\begin{array}{l}\text { c.546C }>\mathrm{T} \\
\text { exon } 3\end{array}$ & $\begin{array}{l}\text { c.668T }>C \\
\text { exon } 3\end{array}$ \\
\hline Ensembl equine sequence & G & $\mathrm{N}$ & C & $\mathrm{T}$ \\
\hline Poitou 1 & $G / G$ & del/del & $T / T$ & $C / C$ \\
\hline Poitou 2 & $G / G$ & del/del & $T / T$ & $C / C$ \\
\hline Poitou 3 & $G / G$ & del/del & $T / T$ & $C / C$ \\
\hline Berry Black 1 & $G / G$ & $N / N$ & $T / T$ & $C / C$ \\
\hline Berry Black 2 & $G / A$ & $N / N$ & $T / T$ & $C / C$ \\
\hline Consequence & p.W82* & p.M145Vfs*15 & p. $(=)$ & p.L223S \\
\hline PolyPhen-2 prediction & & & & Benign \\
\hline
\end{tabular}

$\mathrm{N}$ : no deletion. del: AT deletion.

c.433_434delAT deletion. Among the 35 long-haired Poitou donkeys, 31 were homozygous for the FGF5 mutated allele containing the c.433_434delAT deletion, while four Poitou, two Pyrenean, one Normand and one Bourbonnais donkeys were heterozygous. The remaining 63 short-haired donkeys were homozygous for the wild type reference allele (Table 2). Thus, we genotyped 131 shorthaired ponies and horses from nine breeds but did not identify the deletion in this panel (Table 2), which supports the association (chi-square test, $\mathrm{p}=2.62 \times 10^{-20}$ ) between the Poitou deletion and the long-haired phenotype of this breed.

To check whether the four Poitou individuals that were identified as heterozygous for the deletion were compound heterozygotes, we determined the genotypes of the cohort at the c.245G > A SNP. Indeed the four c.433_434delAT heterozygous Poitou individuals were also heterozygous for the c.245G > A nonsense mutation (Table 3). Ninety-three donkeys were homozygous for the wild type reference allele, and interestingly the five remaining donkeys from the cohort (one Berry Black, one Normand, one Pyrenean and two Provence) carried a copy of the mutant allele (Table 3).

Table 2 Genotypes of donkeys and horses for the c.433_434delAT deletion

\begin{tabular}{lllll}
\hline & N/N & N/del & del/del & Total \\
\hline Pyrenean donkeys & 11 & $\mathbf{2}$ & 0 & 13 \\
Provence donkeys & 16 & 0 & 0 & 16 \\
Normand donkeys & 17 & $\mathbf{1}$ & 0 & 18 \\
Berry Black donkeys & 8 & 0 & 0 & 8 \\
Cotentin donkeys & 10 & 0 & 0 & 10 \\
Bourbonnais donkeys & 1 & $\mathbf{1}$ & 0 & 2 \\
Poitou donkeys & 0 & $\mathbf{4}$ & $\mathbf{3 1}$ & 35 \\
Total in donkeys & 63 & $\mathbf{8}$ & $\mathbf{3 1}$ & 102 \\
Horses and ponies from nine breeds & 131 & 0 & 0 & 131 \\
\hline *These four Poitou donkeys are the same animals & as those shown in italics in
\end{tabular}

*These four Poitou donkeys are the same animals as those shown in italics in Table 3; N: no deletion; del: AT deletion. Heterozygous and homozygous mutant animals are bolded.
As stated above, the c.433_434delAT deletion induces a frameshift that leads to a premature stop codon at position 159 in the protein. To evaluate the putative functional impact of the c.433_434delAT deletion on FGF5, 3D models were built using the Phyre server [18] and five human FGF proteins as templates. The models predicted that the truncated protein lacked seven of the $12 \beta$-strands that define the canonical FGF trefoil (Figure 2; [23]), which supports the hypothesis that the c.433_434delAT deletion can impair FGF5 function. Similarly, the models predicted a loss-of-function for the shorter, truncated protein produced from the c.245G > A allele and which lacked all $\beta$-strands (Figure 2).

To conclude, we propose that the prevalent c.433_ 434delAT deletion identified in all Poitou donkeys constitutes the recessive long-hair FGF5 $5^{\text {l-poitou }}$ Poitou allele and that the c.245G > A nonsense mutation, which is rarely identified in the Poitou and other breeds, constitutes a long-hair FGF5 $5^{\text {-donkey }}$ donkey allele.

\section{Discussion}

Although the long-hair phenotype is a distinctive characteristic of the Poitou donkey breed, the molecular mechanisms responsible for this trait have not yet been reported. Since the mapping tools that have been developed for

Table 3 Genotypes of donkeys for the c.245G > A SNP

\begin{tabular}{lllll}
\hline & G/G & $\boldsymbol{G} / \boldsymbol{A}$ & $\boldsymbol{A} / \boldsymbol{A}$ & Total \\
\hline Pyrenean donkeys & 12 & $\mathbf{1}$ & 0 & 13 \\
Provence donkeys & 14 & $\mathbf{2}$ & 0 & 16 \\
Normand donkeys & 17 & $\mathbf{1}$ & 0 & 18 \\
Berry Black donkeys & 7 & $\mathbf{1}$ & 0 & 8 \\
Cotentin donkeys & 10 & 0 & 0 & 10 \\
Bourbonnais donkeys & 2 & 0 & 0 & 2 \\
Poitou donkeys & 31 & $\mathbf{4}^{*}$ & 0 & 35 \\
Total & 93 & 9 & 0 & 102 \\
\hline
\end{tabular}

*These four Poitou donkeys are the same animals as those shown in italics in Table 2. Heterozygous animals are bolded. 

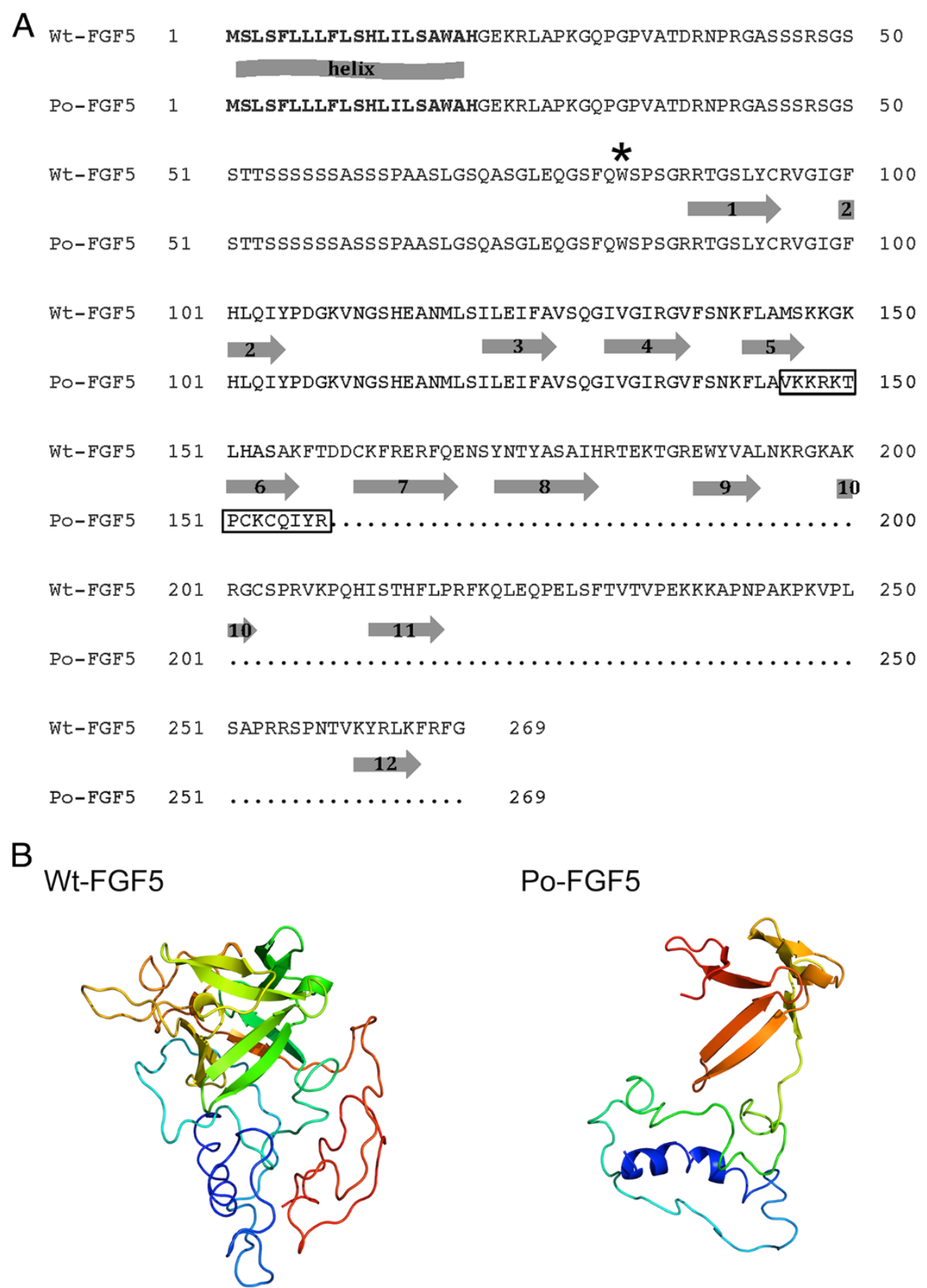

Figure 2 Wild type and mutant FGF5 proteins. (A) Alignment of the FGF5 amino-acid sequence predicted from the equine wild type allele (Wt-FGF5, [Ensembl:ENSECAT00000016512]) and the c.433_434delAT mutant Poitou allele (Po-FGF5). The signal peptide is indicated in bold characters. The secondary structure predicted by the Phyre ${ }^{2}$ program is represented by the arrows below the Wt-FGF5 sequence. Arrows with numbers represent the $12 \beta$-strands. The predicted $\mathrm{N}$-ter $\mathrm{a}$-helix is indicated. The frameshift produced by the c.433_434delAT deletion is framed. A star indicates the location of the stop codon produced by the c.245G > A nonsense mutation. (B) 3D models built using the Phyre server represent the horse wild type Wt-FGF5 (left) and the donkey c.433_434delAT Poitou Po-FGF5 (right) proteins; the a-helix is highlighted in blue and the $\beta$ strands are depicted by arrows.

horses are poorly efficient in donkeys [22], we opted for a candidate gene approach that targeted the FGF5 gene for which loss-of-function mutations have been shown to produce a conserved long-hair phenotype in mammals (http://omia.angis.org.au). Due to the high conservation between donkey sequences and the horse reference genome, we successfully amplified the complete FGF5 coding sequence in donkeys using intron primers that were designed from the horse sequence [21]. Among the polymorphisms identified, the Poitou-specific c.433_434delAT 
deletion was postulated to be a deleterious mutation, since the resulting allele produces a truncated protein that lacks seven of the $12 \beta$-strands involved in FGF5 function. Indeed, if this allele is present in two copies, it is associated with the long-hair phenotype in $\sim 90 \%(31 / 35)$ of the Poitou donkeys from our cohort. The remaining $10 \%$ of longhaired Poitou donkeys from the cohort could be explained by the combined inheritance of this c.433_434delAT deletion with another recessive c. $245 \mathrm{G}>\mathrm{A}$ mutation. Interestingly, the latter mutation was also detected as a single copy in individuals from four other short-hair donkey breeds.

We observed high levels of similarities between the donkey FGF5 protein sequence and several mammalian orthologs, which emphasizes that the wild type FGF5 protein may also be functional in donkeys. FGF ligands carry out their diverse functions by binding and activating FGF receptors (FGFR), which belong to the family of tyrosine kinase receptors, in a heparan sulphate glycosaminoglycan (HSGAG)-dependent manner [2,23,24]. Thus, binding of the FGF ligand to HSGAG and FGFR promotes dimerization and stabilization of the FGF-HSGAGFGFR complex, which induces the activation of tyrosine kinase and the downstream intracellular signaling processes [24]. While the HSGAG binding site of FGF is composed of the $\beta 1-\beta 2$ loop and parts of the region spanning $\beta 10$ and $\beta 12$ [2], residues involved in the FGFR interaction are located in several regions including $\beta 1, \beta 2, \beta 9$ and $\beta 12$ strands, $\beta 1-\beta 2$ and $\beta 8-\beta 9$ turns, $\beta 3-\beta 4$ loop for the FGF - D2 region of FGFR and in regions including $\beta 1$ and $\beta 4$ to $\beta 8$ sheets and $\beta 4-\beta 5, \beta 7-\beta 8$ and $\beta 8-\beta 9$ loops for the FGF - D3 region of FGFR [23].

It should be noted that the $12 \beta$-strands and critical residues involved in the binding of FGF5 with HSGAG and FGFR interactions are conserved in horses and donkeys (Figure 2 and [23]), which suggests that the wild type FGF5 protein is functional in both species. In silico, the c.245G > A and the c.433_434delAT mutant alleles yield truncated FGF5 proteins that lack all or the last seven $\beta$-strands, respectively (Figure 2). If correctly addressed within the cell and secreted, the encoded truncated proteins are thus unlikely to mediate the FGF5 signaling process, which strongly suggests that $F G F 5^{\text {-poitou }}$ and $F G F 5^{l-d o n k e y}$ are two loss-of-function donkey alleles of FGF5. This is in agreement with long-haired phenotypes previously observed in FGF5-deficient mice, humans, dogs and cats [4,7-12].

To the best of our knowledge, long-haired horses have never been reported. In contrast, four long-haired donkey breeds have been described, including the Poitou breed. For this breed, we provide strong evidence that all individuals are deficient in FGF5 signaling, either because they carry two copies of the most prevalent loss-offunction $F G F 5^{\text {l-poitou }}$ allele, or because they are compound heterozygotes carrying the two loss-of-function FGF5 $5^{\text {-poitou }}$ and $F G F 5^{l-d o n k e y}$ alleles. Interestingly, these two alleles were also detected at low frequency in heterozygous individuals from short-haired French breeds. The probability that the two FGF5 $5^{\text {-poitou }}$ and FGF5 $5^{\text {-donkey }}$ alleles could have independently arisen in several breeds as a consequence of distinct mutation events is very low. It is more likely that they result from founder mutations that were disseminated because of outcrossing. Altough the genealogy of the donkeys included in this study is not known, the hypothesis of common ancestors is supported by the history of French breeds. Indeed, the studbooks of Berry Black, Normand and Bourbonnais breeds are very recent, i.e. they were created in 1994, 1997 and 2002, respectively. In 2011, 34 Berry Black and 66 Normand donkeys were registered in France, and in 2012, 10 Bourbonnais donkeys were registered (http://www.haras-nationaux.fr). Previous registrations were accepted based on aesthetic criteria, which has certainly favored individuals with genomic admixture of other breeds.

Other long-haired donkeys belong to the American miniature donkey breed that displays the so-called "woolly phenotype", and to the two endangered, related Spanish Zamorano-Leonés and Portuguese Burro de Miranda breeds. In the 1990s', Zamorano jennies were imported to the French Poitou region during a preservation program, and, in the last decades, many Spanish donkeys were imported to France. Thus, it can be hypothesized that the $F G F 5^{\text {l-donkey }}$ allele was introgressed from the Spanish breeding stock, which is supported by genealogical analyses that revealed that the four Poitou compound heterozygotes of our cohort were descendants of Zamorano jennies. Moreover, it is highly problable that the most prevalent FGF5 ${ }^{\text {l-poitou }}$ allele was fixed by Poitou breeders during the $19^{\text {th }}$ century, which corresponded to the golden period of this breed. Haplotype analyses on a larger cohort of donkeys, including Miranda and Zamorano individuals, would help confirm these hypotheses. Currently, the small number of registered donkeys in each breed prevents more in-depth analyses.

\section{Conclusions}

Our results highlight the allelic heterogeneity of the longhair phenotype in donkeys and enlarge the panel of recessive FGF5 loss-of-function alleles described in mammals. Identification of mutations that underlie the long-hair phenotype in donkeys will help breeders to detect heterozygous carriers in short-haired French breeds and thus prevent matings at risk to produce non-eligible longhaired foals. Finally, it would be interesting to test for the FGF5 $5^{\text {-poitou }}$ and FGF5 $5^{\text {l-donkey }}$ alleles the American miniature donkeys that have been reported to be woolly-haired and that were produced in the USA. The DNA test developed from this study is available to the community and will help breeders to identify carriers in their breeding stocks. 


\section{Additional file}

Additional file 1: Table S1. PCR and sequencing primers. The data provided represent the sequences and annealing temperatures for the primers that were used to amplify and sequence the three FGF5 exons. Table S2. Genotyping primers. The data provided represent the two sets of three primers that were used to amplify and pyrosequence the two FGF5 mutations identified in donkeys.

\section{Competing interests}

The authors declare that they have no competing interests.

\section{Authors' contributions}

MA designed the study and collected samples. RL and MA performed the experiments. MA and LT wrote the manuscript. All authors read and approved the final manuscript.

\section{Acknowledgements}

Authors wish to thank owners and breeders for providing samples and pictures and Jean-Claude Mériaux from Labogena for providing DNA samples from ponies and horses. This project was funded by the French National Donkey Institute (INAM, Institut National Asin et Mulassier, France).

Received: 15 April 2014 Accepted: 14 September 2014

Published online: 25 September 2014

\section{References}

1. Ozawa K, Suzuki S, Asada M, Tomooka Y, Li AJ, Yoneda A, Komi A, Imamura $\mathrm{T}$ : An alternatively spliced fibroblast growth factor (FGF)-5 mRNA is abundant in brain and translates into a partial agonist/antagonist for FGF-5 neurotrophic activity. J Biol Chem 1998, 273:29262-29271.

2. Beenken A, Mohammadi M: The FGF family: biology, pathophysiology and therapy. Nat Rev Drug Discov 2009, 8:235-253.

3. Zhan X, Bates B, Hu XG, Goldfarb M: The human FGF-5 oncogene encodes a novel protein related to fibroblast growth factors. Mol Cell Biol 1988, 8:3487-3495.

4. Hébert JM, Rosenquist T, Götz J, Martin GR: FGF5 as a regulator of the hair growth cycle: evidence from targeted and spontaneous mutations. Cell 1994, 78:1017-1025.

5. Suzuki S, Ota Y, Ozawa K, Imamura T: Dual-mode regulation of hair growth cycle by two Fgf-5 gene products. J Invest Dermatol 2000, 114:456-463.

6. Hattori Y, Yamasaki M, Itoh N: The rat FGF-5 mRNA variant generated by alternative splicing encodes a novel truncated form of FGF-5. Biochim Biophys Acta 1996, 1306:31-33.

7. Sundberg JP, Rourk MH, Boggess D, Hogan ME, Sundberg BA, Bertolino AP: Angora mouse mutation: altered hair cycle, follicular dystrophy, phenotypic maintenance of skin grafts, and changes in keratin expression. Vet Pathol 1997, 34:171-179.

8. Mizuno S, lijima S, Okano T, Kajiwara N, Kunita S, Sugiyama F, Yagami K: Retrotransposon-mediated Fgf5(go-Utr) mutant mice with long pelage hair. Exp Anim 2011, 60:161-167.

9. Housley DJ, Venta PJ: The long and the short of it: evidence that FGF5 is a major determinant of canine 'hair'-itability. Anim Genet 2006, 37:309-315.

10. Dierks C, Mömke S, Philipp U, Distl O: Allelic heterogeneity of FGF5 mutations causes the long-hair phenotype in dogs. Anim Genet 2013, 44:425-431.

11. Kehler JS, David VA, Schäffer AA, Bajema K, Eizirik E, Ryugo DK, Hannah SS, O'Brien SJ, Menotti-Raymond M: Four independent mutations in the feline fibroblast growth factor 5 gene determine the long-haired phenotype in domestic cats. J Hered 2007, 98:555-566.

12. Drögemüller $C$, Rüfenacht $S$, Wichert $B$, Leeb T: Mutations within the FGF5 gene are associated with hair length in cats. Anim Genet 2007, 38:218-221.

13. Orlando L, Ginolhac A, Zhang G, Froese D, Albrechtsen A, Stiller M, Schubert M, Cappellini E, Petersen B, Moltke I, Johnson PL, Fumagalli M, Vilstrup JT, Raghavan M, Korneliussen T, Malaspinas AS, Vogt J, Szklarczyk D, Kelstrup CD, Vinther J, Dolocan A, Stenderup J, Velazquez AM, Cahill J, Rasmussen M, Wang X, Min J, Zazula GD, Seguin-Orlando A, Mortensen C: Recalibrating Equus evolution using the genome sequence of an early Middle Pleistocene horse. Nature 2013, 499:74-78.
14. Untergasser A, Cutcutache I, Koressaar T, Ye J, Faircloth BC, Remm M, Rozen SG: Primer3-new capabilities and interfaces. Nucleic Acids Res 2012, 40:e115.

15. Corpet F: Multiple sequence alignment with hierarchical clustering. Nucleic Acids Res 1988, 16:10881-10890.

16. Ahmadian A, Gharizadeh B, Gustafsson AC, Sterky F, Nyrén P, Uhlén M, Lundeberg J: Single-nucleotide polymorphism analysis by pyrosequencing. Anal Biochem 2000, 280:103-110.

17. Adzhubei IA, Schmidt S, Peshkin L, Ramensky VE, Gerasimova A, Bork P Kondrashov AS, Sunyaev SR: A method and server for predicting damaging missense mutations. Nat Methods 2010, 7:248-249.

18. Kelley LA, Sternberg MJ: Protein structure prediction on the Web: a case study using the Phyre server. Nat Protoc 2009, 4:363-371.

19. Musilova P, Kubickova S, Vahala J, Rubes J: Subchromosomal karyotype evolution in Equidae. Chromos Res 2013, 21:175-187.

20. Vidale P, Piras FM, Nergadze SG, Bertoni L, Verini-Supplizi A, Adelson D, Guérin G, Giulotto E: Chromosomal assignment of six genes (EIF4G3, HSP90, RBBP6, IL8, TERT, and TERC) in four species of the genus Equus. Anim Biotechnol 2011, 22:119-123.

21. Wade CM, Giulotto E, Sigurdsson S, Zoli M, Gnerre S, Imsland F, Lear TL, Adelson DL, Bailey E, Bellone RR, Blöcker H, Distl O, Edgar RC, Garber M, Leeb T, Mauceli E, MacLeod JN, Penedo MC, Raison JM, Sharpe T, Vogel J, Andersson L, Antczak DF, Biagi T, Binns MM, Chowdhary BP, Coleman SJ, Della Valle G, Fryc S, Guérin G: Genome sequence, comparative analysis, and population genetics of the domestic horse. Science 2009, 326:865-867.

22. McCue ME, Bannasch DL, Petersen JL, Gurr J, Bailey E, Binns MM, Distl O, Guerin G, Hasegawa T, Hill EW, Leeb T, Lindgren G, Penedo MC, Røed KH, Ryder OA, Swinburne JE, Tozaki T, Valberg SJ, Vaudin M, Lindblad-Toh K, Wade CM, Mickelson JR: A high density SNP array for the domestic horse and extant Perissodactyla: utility for association mapping, genetic diversity, and phylogeny studies. PLoS Genet 2012, 8:e1002451.

23. Mohammadi M, Olsen SK, Ibrahimi OA: Structural basis for fibroblast growth factor receptor activation. Cytokine Growth Factor Rev 2005, 16:107-137.

24. Goetz R, Mohammadi M: Exploring mechanisms of FGF signalling through the lens of structural biology. Nat Rev Mol Cell Biol 2013, 14:166-180.

doi:10.1186/s12711-014-0065-5

Cite this article as: Legrand et al:: Two recessive mutations in FGF5 are associated with the long-hair phenotype in donkeys. Genetics Selection Evolution 2014 46:65

\section{Submit your next manuscript to BioMed Central and take full advantage of:}

- Convenient online submission

- Thorough peer review

- No space constraints or color figure charges

- Immediate publication on acceptance

- Inclusion in PubMed, CAS, Scopus and Google Scholar

- Research which is freely available for redistribution 